\title{
Assessment of Contrast Sensitivity in Age Related Maculopathy Simulation
}

\author{
Tzamouranis Dorotheos-Dimitrios, BSc, MSc. \\ Kakoura Stavroula, BSc, MSc. \\ Karetsos George, BSc, MSc.
}

Investigative Techniques in Optometry Research Group

Department of Biomedical Sciences

School of Health and Welfare

University of West Attica

Campus One, Athens, Greece

\section{Chandrinos Aristeidis, BSc, MPhil, PhD.}

\author{
Assistant Professor of Optometry \\ Department of Biomedical Sciences \\ School of Health and Welfare \\ University of West Attica \\ Campus One, Athens, Greece
}

\begin{abstract}
Contrast sensitivity (CS) discriminates objects in low contrast levels, while visual acuity measures how big an object must be to be seen. In pathological eye conditions, numerous studies have noted the importance of CS, in early diagnosis. Age-related macular degeneration (AMD) is a progressive retinal disease and a common cause of blindness for patients over 50's that cannot be detected only by visual acuity. Although various studies described the impact of $A M D$ on psychophysical daily activities, the quality of life is defined as the general well-being and health of individuals. Current research aims to revise the CS test as diagnostic and predictive clinical tool of visual function by simulating AMD. Statistical analysis of the results, demonstrated statistically significant decline from Normal to Early, Medium and Advanced stages of simulated disease, but no significant difference between right and left eyes or between genders. The assessment of contrast sensitivity can be a part of medical preventive eye care, furthermore for populations with limited access to public health.
\end{abstract}

Keywords: Contrast Sensitivity (CS), Age-related Macular Degeneration (AMD), Pelli Robson chart, Simulation, AMD clinical tools, Quality of life.

\section{Introduction}

The quality of vision is determined by three factors, the clarity of the image, the size of the point image and the perception of the image by the photoreceptors in the retina.

Visual acuity is the ability of the eye to distinguish, two points in space as separate. In young eyes the average visual acuity is maximum 20/20, while in elderly subjects, over 60 years old, visual acuity decreases to a lower level (Elliot et al., 1995). Clinically, visual acuity (VA), is evaluated with optotypes or visual acuity charts. Contrast Sensitivity (CS) is a psychophysical assessment of the quality of vision and is required to differentiate a figure from its background. It is based on modulation transfer function, which usually vary in accordance to spatial frequency. The Contrast sensitivity is defined as the inverse of the minimum contrast ( $\mathrm{Cmin})$, that is $\mathrm{CS}=1$ / Cmin. A contrast sensitivity test typically contains minimum light contrast for sinusoidal gratings and frequencies between 0.5 and $20 \mathrm{c} / \mathrm{deg}$. Spatial frequencies express the characteristics of an optical system. High frequencies correspond to lower brightness contrasts while low frequencies correspond to higher contrast.

Contrast sensitivity is decreased in clinical conditions and the maximum contrast sensitivity could be reduced even when the visual acuity is normal. The contrast sensitivity is frequently reduced in pathological conditions, such as myopia (Collins \& Carney, 1990), glow (Abrahamson \& Sjöstrand, 1986), cataracts (Hess \& Woo, 1978), amblyopia (Freedman \& Thibos, et al., 1988), ocular hypertension (Gandolfi, 2005), glaucoma (Stamper, 1984) and dry eye (Rolando et al., 1998) (Bodis-Wollner, 1972), multiple sclerosis (Regan et al., 1981), Parkinson's disease (BodisWollner \& Onofrj, 1986) and schizophrenia (Cimmer et al., 2006). 
In addition, loss of contrast sensitivity is a common side effect of many prescription medicines (Li, Tripathi \& Tripathi, 2008; Santaella \& Fraunfelder, 2007). Age-related macular degeneration (AMD) is a common condition of vision loss, etiologically complex disease with no cure so far, with disease development strongly influenced by environmental and genetic factors (Lim et al., 2012 ; Fritsche et al., 2014; DeAngelis et al., 2017). Two stages are known for this disease: early AMD, (the dry form, likely due to progressive macular cell loss), which is characterized by drusen and pigmentary changes, and late AMD (the wet form), which can be distinguished in 2 subtypes-geographic atrophy (GA) and choroidal neo-vascularization (CNV) (Kanski, 2004; De Jong, 2006; Colijn et al., 2017). At a very early stage, there are no signs of vision loss. At a fairly advanced stage, deprives an individual of his or her ability to perform basic daily activities, such as reading, recognizing faces or driving a car, as a result of a central scotoma usually being present.

Nowadays, more or less of 11 million individuals are affected by AMD in the United States (U.S.) alone, with a global prevalence of 170 million. AMD is thereby the leading cause of visual disability in the industrialized world and the third leading cause globally (Pascolini, 2012; Bright Focus Foundation, 2015). Aging is the greatest risk factor therefore, the prevalence of AMD in the U.S. is anticipated to increase to 22 million by the year 2050, while the global prevalence is expected to increase to 288 million by the year 2040 (Wong, 2014; Bright Focus Foundation, 2015).

In the U.S., the prevalence of AMD is similar to that of all invasive cancers combined and more than double the prevalence of Alzheimer's Disease. This high prevalence leads to an annual $\$ 4.6$ billion direct healthcare cost due to AMD in the U.S. (Bright Focus Foundation, 2015 \& Prevent blindness, 2015). As the aging population increases, this expenditure is likewise expected to increase proportionately.

An Australian study in 2004, states that loss of driving ability is an important factor in losing independence as patients have to rely on others to move around, which may lead to social isolation, a factor of the progressive development of depression. The study also emphasized, that people resist asking for help in the fear that they will become heavy and make a big effort to remain independent, (Wong, 2004). Loss of independence may be such that residential care is required. The loss of vision due to AMD may lead to additional living costs for helping patients with a higher visual acuity loss according to a French study (Bonastre, 2003). Symptoms such as the above, as well as the lack of expectation for improvement, compose an psychological distress and push patients in to extensive activities restriction and then social exclusion.

\subsection{The Objectives of the Study}

This study investigates and compares the effect on Contrast Sensitivity of simulated macular degeneration in healthy individuals and in different stage of disease severity. Furthermore, considers a possible AMD effect on patients at every day activities, based on the progression rate of the disease. In addition, this study tries to assess contrast sensitivity as a tool for prognosis and identification of the early stages of the AMD disease.

\section{Methodology}

\subsection{Research Design}

The research was conducted at the Optometry Clinic of the University of Western Attica, in Athens, Greece. The participants underwent an ophthalmic assessment, which included complete medical history and visual acuity assessment, refraction, binocular vision assessment, pupillary reflection and diameter, slit-lamp evaluation of the anterior eye, and indirect ophthalmoscopy of the macula. The sample was carefully selected among a healthy group of 30 individuals aged 18 to 35 years old, following specific exclusion and inclusion criteria. All tests for every individual performed in scotopic room conditions $(0,003 \mathrm{Lux}$ and $0,001 \mathrm{Cd} / \mathrm{m} 2$ at the pupil level).The luminance was measured by digital camera.

The visual acuity was assessed on a Baley-Lovie illuminated chart (auto-zoom) mounted at $4 \mathrm{~m}$ distance. The visual acuity was verified by electronic refractometer, phoropter, a pinhole test, a complete spherical correction, Jackson cross cylinder test and polarized filters to equalize binocular vision. The examination started with the right eye, followed by the left eye and at last binocular. Next the procedure of lenses simulation took place by producing controlled scaled central scotoma to all three pairs for every stage and each individual.

For this purpose were used, "3M Over-the-Glass eyewear" to be worn over prescription eyewear. These frames were made of Clear polycarbonate lenses and scratch-resistant coatings, easy to clean with soap and water, providing the ability to reuse it for more participants with no impact to the lenses visibility. The contrast sensitivity was assessed by an original UK Pelli-Robson chart, mounted at a distance of 3 metres and in photopic room conditions of 162 Lux and $48,6 \mathrm{Cd} / \mathrm{m} 2$ at the pupil level. 


\subsection{Respondents and Sampling Plan}

Exclusion criteria were the presence of astigmatism, previous eye injuries or other pathological conditions, hereditary diseases (diabetes, glaucoma, etc.), any presence of phoria, strabismus or diplopia

Inclusion criteria included emmetropia or ametropia accepted maximum refractive error \pm 2.00 dpts, but always at least 6/6 VA after correction. Although recruited 30 individuals after the exclusion criteria the evaluated sample comprised by 12 individuals, 6 men and 6 women (Tables 1, 2).

Table 1: Distribution of age and gender for 12 individuals of the sample.

\begin{tabular}{|c|c|c|}
\hline Age & \multicolumn{2}{|c|}{ Gender } \\
\hline & Male & Female \\
\hline $18-24$ & 2 & 3 \\
\hline $25-30$ & 4 & 3 \\
\hline
\end{tabular}

Table 2: Refractive errors within acceptable restrictions Refractive error

\begin{tabular}{|c|c|c|c|c|c|c|}
\hline Gender & $\mathrm{R}-2,00$ & $\mathrm{R}-0,50$ & $\mathrm{R}$ PLANO & $\mathrm{L}-2,00$ & $\mathrm{~L}-0,50$ & L PLANO \\
\hline Male & 2 & 3 & 1 & 2 & 3 & 1 \\
\hline Female & 3 & 1 & 2 & 3 & 1 & 2 \\
\hline
\end{tabular}

\subsection{Instrument and Data Gathering Procedure}

Subsequently, created controlled luminance conditions, simulating the natural light (162 lux) using a light source (projector), a dimmer switch and a digital luxmeter. The white sensor of the luxmeter, was facing the lighting source, at a constant position ( 3 meters distance in order to correspond to spatial frequencies of 3 cycles / degree) equal to the distance between the Pelli Robson chart and the individual for every test.

The testing room had been completely darkened before each measurement. Before every second test, each individual was moved to a dark room with full eye coverage, using a sleeping mask, staying there for about 20 minutes, in order to avoid the fatigue effect during the next contrast sensitivity assessment. At the end of the resting period, the mask was removed and the brightness of the testing room was gradually increased to avoid blurring after a sudden brightness change.

An original UK Pelli-Robson chart, mounted at a distance of 3 metres, was used. The chart with dimensions $90 \times 60 \mathrm{~cm}$ (36x24 inches) consisted of 8 lines of letters having different contrast. Each row includes 6 letters consisting of two triplets. The contrast decreases downwards from row to row and horizontally from right to left triplet in each row. The font size is $4.9 \times 4.9 \mathrm{~mm}(2 \times 2$ inches). The first three letters of the left triplet at first row have the maximum contrast (contrast $=1$ ), and the letters of the last left triplet at the lower row have the minimum contrast (contrast $=0.006$ ). Contrast sensitivity is defined as the inverse analogue of contrast (CS =1/ contrast) (Maija, 2001). Each triplet is calibrated with $0.15 \log$ contrast sensitivity and consequently each letter of every triplet, corresponds to $0.05 \log$ contrast sensitivity (Fig.1).

\section{PELLI - ROBSON}

\begin{tabular}{|c|c|c|c|c|c|c|c|}
\hline 0.00 & $H$ & 5 & $z$ & $\mathbf{D}$ & $\mathbf{S}$ & $\mathbf{N}$ & 0.15 \\
\hline 0.30 & $\mathbf{0}$ & $\mathbf{K}$ & $\mathbf{R}$ & $z$ & $\mathbf{v}$ & $\mathbf{R}$ & 0.45 \\
\hline 0.60 & $N$ & $\mathbf{D}$ & $\mathbf{c}$ & 0 & 5 & $\mathbf{K}$ & 0.75 \\
\hline 0.90 & 0 & z & $\mathbf{K}$ & $\mathbf{v}$ & $\mathrm{H}$ & $\mathbf{z}$ & 1.05 \\
\hline 1.20 & $\mathbf{N}$ & $H$ & 0 & $\mathbf{N}$ & $\mathbf{R}$ & $\mathbf{D}$ & 1.35 \\
\hline 1.50 & $\mathbf{V}$ & $\boldsymbol{R}$ & 0 & 0 & $\mathbf{V}$ & $H$ & 1.65 \\
\hline 1.80 & c & D & 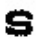 & $\mathbf{N}$ & $\mathbf{D}$ & & 1.95 \\
\hline 2.10 & $\mathbf{K}$ & $V$ & 7 & 0 & $H$ & $\mathbf{R}$ & 2.25 \\
\hline
\end{tabular}

Fig. 1: Pelli -Robson chart, consisting of 8 rows of different contrast letters and 16 triplets. The score numbers correspond to the far left and far right letters of every row. 
Initially, the measured values of normal individuals without simulation were recorded and then the values in the simulation stages one by one (Early, Medium and Advanced). The face measurements, pupil distances (horizontally and perpendicular), and pupil diameter determined the position and the size of the grey colour simulated central scotoma, for every specific stage of the disease (Early stage: $0,5 \mathrm{~mm}$ in central area, Medium: $1 \mathrm{~mm}$, and Advanced: 1,5 $\mathrm{mm}$ in diameter). Personal pairs of simulated glasses were used for each individual and for all the three levels of disease, after the US pattern modification (US Patent 5,737,056, Martin et al., 1998) entitled "Methods for simulations of visual disabilities" for contact lenses, ophthalmic lenses and a pair of eyeglasses.

According to the examination procedure, the subject was asked to read the letters for every row, from left to right and from the upper to the lower row. At the first reading failure the test was completed and the sensitivity score according to Fig. 2 was recorded. For greater accuracy, the recorded results referred to each letter $(0.05 \mathrm{log})$ of the triplet and not to the entire triplet $(0.15 \mathrm{log})$ as defined by the designers of Pelli-Robson chart (Pelli et al. 1988). Reading letter $\mathbf{C}$ as an $\mathbf{O}$ was considered as failure of reading the letter. Elliot (2006), accepted the reading of the letter $\mathrm{C}$ as an $\mathrm{O}$ as a correct response to further balance the legibility of the letters. Contrast sensitivity was recorded, always testing first the right eye and covered the left, then testing the left eye and covered the right eye. Finally tested binocular for every simulation stage.

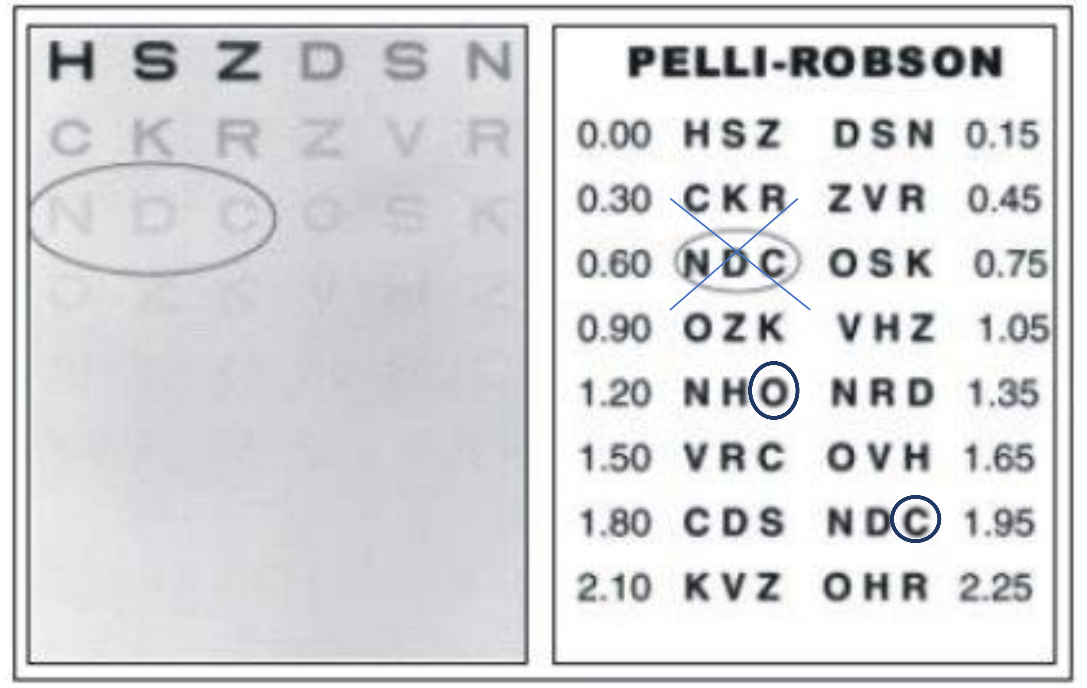

Figure 2. For greater accuracy, the recorded results referred to each letter $(0.05 \log )$ of the triplet and not to the entire triplet $(0.15 \mathrm{log})$.

\section{Results}

The table below, includes contrast sensitivity values during the Pelli-Robson examination test for male and female individuals for right eyes, left eyes and binocular, in AMD simulation conditions for all stages (normal, early, medium, late), (Table 3). In normal stage CS values recorded without simulation. For the male individuals, contrast sensitivity values in right eyes ranged from $1.75 \log$ for the normal to $0.45 \log$ for late AMD stage, for left eyes CS values ranged from $1.7 \log$ for the normal to $0.5 \log$ for the late, and in binocular test, values ranged from $1.63 \log$ for the normal to $0.40 \log$ for the late stage. Correspondingly, for the female individuals, in right eyes contrast sensitivity values ranged from $1.65 \log$ for the normal to $0.25 \log$ for the late stage, in left eyes CS values ranged from $1.7 \log$ for the normal to $0.50 \log$ for the late, and the binocular CS values ranged from $2 \log$ in the normal stage to $0.2 \log$ in the advanced AMD stage.

Table 3: Pelli-Robson contrast sensitivity values $(\log )$ for right eye, left eye and binocular, for genders. Table includes Mean, Standard Deviation (SD), Median, Min (Max) and Maximum (Max) values, 1st and 3nd quartile ( 25 and $75 \%$ of the normal value respectively) at all stages. Norm $=$ Normal, Early $=$ Early, Medium $=$ Medium, Late $=$ Advanced AMD.

\begin{tabular}{|c|c|c|c|c|}
\hline \multirow[t]{2}{*}{ MALE } & \multicolumn{3}{|c|}{ Right Eye } & \multirow[b]{2}{*}{ Late } \\
\hline & Norm & Early & Medium & \\
\hline Mean & 1,60 & 1,42 & 1,08 & 0,57 \\
\hline SD & 0,10 & 0,12 & 0,14 & 0,14 \\
\hline Median & 1,55 & 1,35 & 1,00 & 0,55 \\
\hline
\end{tabular}

\begin{tabular}{|llll|}
\hline \multicolumn{3}{|c|}{ Left Eye } & \\
\hline Norm & Early & Medium & Late \\
\hline 1,62 & 1,45 & 1,11 & 0,69 \\
\hline 0,08 & 0,14 & 0,21 & 0,20 \\
\hline 1,60 & 1,45 & 1,20 & 0,65 \\
\hline
\end{tabular}




\begin{tabular}{|c|c|c|c|c|c|c|c|c|}
\hline Min & 1,50 & 1,30 & 0,95 & 0,45 & 1,55 & 1,25 & 0,80 & 0,50 \\
\hline Max & 1,75 & 1,55 & 1,25 & 0,80 & 1,70 & 1,60 & 1,30 & 0,90 \\
\hline 1st quartile & 1,55 & 1,35 & 1,00 & 0,45 & 1,55 & 1,40 & 1,00 & 0,50 \\
\hline 3rd quartile & 1,65 & 1,55 & 1,20 & 0,60 & 1,70 & 1,55 & 1,25 & 0,90 \\
\hline
\end{tabular}

\begin{tabular}{|c|c|c|c|c|}
\hline \multirow[t]{2}{*}{ FEMALE } & \multicolumn{3}{|c|}{ RightEye } & \multirow[b]{2}{*}{ Late } \\
\hline & Norm & Early & Medium & \\
\hline Mean & 1,56 & 1,44 & 1,11 & 0,48 \\
\hline SD & 0,09 & 0,13 & 0,13 & 0,14 \\
\hline Median & 1,60 & 1,50 & 1,15 & 0,50 \\
\hline Min & 1,40 & 1,20 & 0,90 & 0,25 \\
\hline Max & 1,65 & 1,55 & 1,25 & 0,65 \\
\hline 1st quartile & 1,53 & 1,40 & 1,03 & 0,40 \\
\hline 3rd quartile & 1,63 & 1,53 & 1,20 & 1,20 \\
\hline
\end{tabular}

\begin{tabular}{|c|c|c|c|}
\hline \multicolumn{4}{|c|}{ Left Eye } \\
\hline Norm & Early & Medium & Late \\
\hline 1,63 & 1,48 & 1,13 & 0,70 \\
\hline 0,11 & 0,11 & 0,18 & 0,28 \\
\hline 1,63 & 1,48 & 1,13 & 0,70 \\
\hline 1,55 & 1,40 & 1,00 & 0,50 \\
\hline 1,70 & 1,55 & 1,25 & 0,90 \\
\hline 1,59 & 1,44 & 1,06 & 0,60 \\
\hline 1,66 & 1,51 & 1,19 & 0,80 \\
\hline
\end{tabular}

\begin{tabular}{|c|c|c|c|c|}
\hline \multirow[t]{2}{*}{ BINOCULAR } & \multicolumn{3}{|c|}{ MALE } & \multirow[b]{2}{*}{ Late } \\
\hline & Norm & Early & Medium & \\
\hline Mean & 1,58 & 1,46 & 1,11 & 0,80 \\
\hline SD & 0,07 & 0,09 & 0,12 & 0,57 \\
\hline Median & 1,58 & 1,46 & 1,11 & 0,80 \\
\hline Min & 1,53 & 1,40 & 1,03 & 0,40 \\
\hline Max & 1,63 & 1,53 & 1,20 & 1,20 \\
\hline 1st quartile & 1,55 & 1,43 & 1,07 & 0,60 \\
\hline 3rd quartile & 1,60 & 1,49 & 1,16 & 1,00 \\
\hline
\end{tabular}

\begin{tabular}{|c|c|c|c|}
\hline & FEM $A$ & & \\
\hline Norm & Early & Medium & Late \\
\hline 1,81 & 1,51 & 1,17 & 0,54 \\
\hline 0,17 & 0,15 & 0,11 & 0,20 \\
\hline 1,90 & 1,55 & 1,20 & 0,60 \\
\hline 1,50 & 1,25 & 1,00 & 0,25 \\
\hline 2,00 & 1,65 & 1,30 & 0,80 \\
\hline 1,73 & 1,45 & 1,10 & 0,40 \\
\hline 1,93 & 1,63 & 1,25 & 0,68 \\
\hline
\end{tabular}

\subsection{Data Analysis}

Data are reported as means and were compared by One way ANOVA (analysis of variance) method followed by post hoc Tukey honestly significant difference (HSD) test using the software SPSS version 24 (Statistical Package for Social Science) with a 0.05 significance level. Additionally, paired-samples t-test was conducted to determine whether the mean difference between paired observations is statistically significantly different from zero. Box plots were used for detecting outliers in this analysis. In order to assess normality, Shapiro-Wilk test and Normal Q-Q Plots, were used.

\subsection{Interpreting the Analysis}

The ANOVA / POST-HOC Tuckey variance analysis was performed, with multiple comparisons between CS groups in AMD simulate mode, right eyes, left eyes and binocular in all the stages, Normal, Early, Medium, Advanced (Tables $4,5,6$ ). Post-hoc tests were conducted in order to confirm the differences between the groups. Post-hoc tests attempted to test the experimental error rate (usually alpha $=0.05$ ) in the same way that the univariate ANOVA was used instead of multiple t-test tests.

A paired-samples t-test was used to determine whether the mean difference between, right and left eyes (Table 7 ) and Men and Women (Table 8), is statistically significantly different from zero. Box plots were used for detecting outliers in every group of participants, left eyes (fig.3), right eyes (fig. 4), binocular (fig.5).

Table 4:Statistically significant differences were observed, in every group, right eye, left eye and binocular values, $p$-value $=0.000<0.05$. Contrast sensitivity score was statistically significantly different between different groups, Right eyes $F(3,44)=181.551, p<0.05$, Left eyes $F(3,44)=132.189, p<0.05$, Binocular $F(3$, 44) $=$ 64.074, $p<0.05$. 


\begin{tabular}{|c|c|c|c|c|c|c|}
\hline & & ANC & & & & \\
\hline & & Sum of Squares & $\mathrm{df}$ & Mean Square & $\mathrm{F}$ & Sig. \\
\hline$\overline{\text { RIGHT }}$ & Between Groups & 8.020 & 3 & 2.673 & 181.551 & .000 \\
\hline & Within Groups & .648 & 44 & .015 & & \\
\hline & Total & 8.668 & 47 & & & \\
\hline LEFT & Between Groups & 9.332 & 3 & 3.111 & 132.189 & .000 \\
\hline & Within Groups & 1.035 & 44 & .024 & & \\
\hline & Total & 10.367 & 47 & & & \\
\hline BINOCULAR & Between Groups & 7.353 & 3 & 2.451 & 64.074 & .000 \\
\hline & Within Groups & 1.683 & 44 & .038 & & \\
\hline & Total & 9.036 & 47 & & & \\
\hline
\end{tabular}

Table 5 : Descriptive measures for CS values, right eye, left eye, and binocular in each category, normal values, early stage values, medium stage values and advanced (late) stage values of AMD simulation.

Descriptives

\begin{tabular}{|c|c|c|c|c|c|c|c|c|c|}
\hline & & & & & & & & & \\
\hline & & & & & & $\begin{array}{l}95 \% \text { Confide } \\
\text { for }\end{array}$ & $\begin{array}{l}\text { e Interval } \\
\text { an }\end{array}$ & & \\
\hline & & $\mathrm{N}$ & Mean & Std. Deviation & Std. Error & $\begin{array}{l}\text { Lower } \\
\text { Bound }\end{array}$ & $\begin{array}{l}\text { Upper } \\
\text { Bound }\end{array}$ & Min & $\operatorname{Max}$ \\
\hline$\overline{\text { RIGHT }}$ & NORMAL & 12 & 1.5792 & .09160 & .02644 & 1.5210 & 1.6374 & 1.40 & 1.75 \\
\hline & EARLY & 12 & 1.4333 & .11934 & .03445 & 1.3575 & 1.5092 & 1.20 & 1.55 \\
\hline & MEDIUM & 12 & 1.0958 & .12515 & .03613 & 1.0163 & 1.1754 & .90 & 1.25 \\
\hline & LATE & 12 & .5167 & .14355 & .04144 & .4255 & .6079 & .25 & .80 \\
\hline & Total & 48 & 1.1563 & .42945 & .06199 & 1.0316 & 1.2809 & .25 & 1.75 \\
\hline LEFT & NORMAL & 12 & 1.5583 & .13953 & .04028 & 1.4697 & 1.6470 & 1.25 & 1.70 \\
\hline & EARLY & 12 & 1.4167 & .14035 & .04051 & 1.3275 & 1.5058 & 1.20 & 1.60 \\
\hline & MEDIUM & 12 & 1.1333 & .25256 & .07291 & .9729 & 1.2938 & .80 & 1.80 \\
\hline & LATE & 12 & .5375 & .22373 & .06459 & .3953 & .6797 & .15 & .90 \\
\hline & Total & 48 & 1.1615 & .43847 & .06329 & 1.0341 & 1.2888 & .15 & 1.80 \\
\hline BINO & NORMAL & 12 & 1.8042 & .13728 & .03963 & 1.7169 & 1.8914 & 1.50 & 2.00 \\
\hline & EARLY & 12 & 1.5208 & .12515 & .03613 & 1.4413 & 1.6004 & 1.25 & 1.65 \\
\hline & MEDIUM & 12 & 1.2083 & .10624 & .03067 & 1.1408 & 1.2758 & 1.00 & 1.35 \\
\hline & LATE & 12 & .6167 & .21985 & .06346 & .4770 & .7564 & .25 & .95 \\
\hline & Total & 48 & 1.2875 & .46966 & .06779 & 1.1511 & 1.4239 & .25 & 2.00 \\
\hline
\end{tabular}


Table 6 :Multiple comparisons between stages statistically significance were observed in all stages $\mathrm{p}=$ 0.000 the same happens in the right eyes $\mathrm{p}=0.026$. In binocular group, between Normal and Early stages there are no statistically significant differences $\mathrm{p}=0.299>0.05$.

\section{Multiple Comparisons}

Tukey HSD

\begin{tabular}{|c|c|c|c|c|c|c|c|}
\hline $\begin{array}{l}\text { Depender } \\
\text { t Variable }\end{array}$ & (I) STAGES & (J) STAGES & $\begin{array}{c}\text { Mean } \\
\text { Difference(I- } \\
\mathrm{J}) \\
\end{array}$ & Std. Error & Sig. & \multicolumn{2}{|c|}{ 95\% Confidence Interval } \\
\hline \multirow[t]{12}{*}{ RIGHT } & \multirow[t]{3}{*}{ NORMAL } & EARLY & $.14583^{*}$ & .04954 & .026 & .0136 & .2781 \\
\hline & & MEDIUM & $.48333^{*}$ & .04954 & .000 & .3511 & .6156 \\
\hline & & LATE & $1.06250^{*}$ & .04954 & .000 & .9302 & 1.1948 \\
\hline & \multirow[t]{3}{*}{ EARLY } & NORMAL & $-.14583^{*}$ & .04954 & .026 & -.2781 & -.0136 \\
\hline & & MEDIUM & $.33750^{*}$ & .04954 & .000 & .2052 & .4698 \\
\hline & & LATE & $.91667^{*}$ & .04954 & .000 & .7844 & 1.0489 \\
\hline & \multirow[t]{3}{*}{ MEDIUM } & NORMAL & $-.48333^{*}$ & .04954 & .000 & -.6156 & -.3511 \\
\hline & & EARLY & $-.33750^{*}$ & .04954 & .000 & -.4698 & -.2052 \\
\hline & & LATE & $.57917^{*}$ & .04954 & .000 & .4469 & .7114 \\
\hline & \multirow[t]{3}{*}{ LATE } & NORMAL & $-1.06250^{*}$ & .04954 & .000 & -1.1948 & -.9302 \\
\hline & & EARLY & $-.91667^{*}$ & .04954 & .000 & -1.0489 & -.7844 \\
\hline & & MEDIUM & $-.57917^{*}$ & .04954 & .000 & -.7114 & -.4469 \\
\hline \multirow[t]{12}{*}{ LEFT } & \multirow[t]{3}{*}{ NORMAL } & EARLY & $.28333^{*}$ & .06263 & .000 & .1161 & .4505 \\
\hline & & MEDIUM & $.59583^{*}$ & .06263 & .000 & .4286 & .7630 \\
\hline & & LATE & $1.18750^{*}$ & .06263 & .000 & 1.0203 & 1.3547 \\
\hline & \multirow[t]{3}{*}{ EARLY } & NORMAL & $-.28333^{*}$ & .06263 & .000 & -.4505 & -.1161 \\
\hline & & MEDIUM & $.31250^{*}$ & .06263 & .000 & .1453 & .4797 \\
\hline & & LATE & $.90417^{*}$ & .06263 & .000 & .7370 & 1.0714 \\
\hline & \multirow[t]{3}{*}{ MEDIUM } & NORMAL & $-.59583^{*}$ & .06263 & .000 & -.7630 & -.4286 \\
\hline & & EARLY & $-.31250^{*}$ & .06263 & .000 & -.4797 & -.1453 \\
\hline & & LATE & $.59167^{*}$ & .06263 & .000 & .4245 & .7589 \\
\hline & \multirow[t]{3}{*}{ LATE } & NORMAL & $-1.18750^{*}$ & .06263 & .000 & -1.3547 & -1.0203 \\
\hline & & EARLY & $-.90417^{*}$ & .06263 & .000 & -1.0714 & -.7370 \\
\hline & & MEDIUM & $-.59167^{*}$ & .06263 & .000 & -.7589 & -.4245 \\
\hline \multirow[t]{8}{*}{ BINO } & \multirow[t]{3}{*}{ NORMAL } & EARLY & .14167 & .07985 & .299 & -.0715 & .3549 \\
\hline & & MEDIUM & $.42500^{*}$ & .07985 & .000 & .2118 & .6382 \\
\hline & & LATE & $1.02083^{*}$ & .07985 & .000 & .8076 & 1.2340 \\
\hline & \multirow[t]{3}{*}{ EARLY } & NORMAL & -.14167 & .07985 & .299 & -.3549 & .0715 \\
\hline & & MEDIUM & $.28333^{*}$ & .07985 & .005 & .0701 & .4965 \\
\hline & & LATE & $.87917^{*}$ & .07985 & .000 & .6660 & 1.0924 \\
\hline & \multirow[t]{2}{*}{ MEDIUM } & NORMAL & $-.42500^{*}$ & .07985 & .000 & -.6382 & -.2118 \\
\hline & & EARLY & $-.28333^{*}$ & .07985 & .005 & -.4965 & -.0701 \\
\hline
\end{tabular}




\begin{tabular}{|l|l|lrrrrr}
\hline & LATE & $.59583^{*}$ & .07985 & .000 & .3826 & .8090 \\
\cline { 3 - 8 } & & NORMAL & $-1.02083^{*}$ & .07985 & .000 & -1.2340 & -.8076 \\
\cline { 3 - 8 } & EARE & $-.87917^{*}$ & .07985 & .000 & -1.0924 & -.6660 \\
& & MEDIUM & $-.59583^{*}$ & .07985 & .000 & -.8090 & -.3826 \\
\hline
\end{tabular}

*The mean difference is significant at the 0.05 level.

Table 7: There was no statistically significant difference between right eye and left eye (means), $p=0.844>0.05$. A paired-samples t-test was used to determine whether there was a statistically significant mean difference between right and left eyes, $(-0,005 \pm 0,1825)$ and statistically significant increase of $-0,005$ (95\% CI, -0.058 to 0,047$), t(47)=-0,198$, $\mathrm{p}=0,844>0,05, \mathrm{~d}=-0.027$

\section{Paired Samples Test}

\begin{tabular}{|c|c|c|c|c|c|c|c|c|}
\hline \multicolumn{6}{|c|}{ Paired Differences } & \multirow[b]{3}{*}{$\mathrm{t}$} & \multirow[b]{3}{*}{$\mathrm{df}$} & \multirow{3}{*}{$\begin{array}{l}\text { Sig. (2- } \\
\text { tailed) }\end{array}$} \\
\hline & \multirow[b]{2}{*}{ Mean } & \multirow{2}{*}{$\begin{array}{c}\text { Std. } \\
\text { Deviation }\end{array}$} & \multirow{2}{*}{$\begin{array}{c}\text { Std. Error } \\
\text { Mean }\end{array}$} & \multicolumn{2}{|c|}{$\begin{array}{l}\text { 95\% Confidence } \\
\text { Interval of the } \\
\text { Difference }\end{array}$} & & & \\
\hline & & & & Lower & Upper & & & \\
\hline $\begin{array}{ll}\text { Pair } 1 & \text { Right - Left }\end{array}$ & -.00521 & .18255 & .02635 & -.05821 & .04780 & -.198 & 47 & .844 \\
\hline
\end{tabular}

Table 8 :There was no statistically significant difference between male (M) and female (F) (means), $\mathrm{p}=0.059>0.05$. A paired-samples t-test was used to determine whether there was a statistically significant mean difference between Male and Female, $(0,045 \pm 0,113)$ and a statistically significant increase of 0.045 (95\% CI, -0.001 to 0,093$), t(23)=$ $1.984, \mathrm{p}=0,059>0,05, \mathrm{~d}=0.39$

\section{Paired Samples Test}
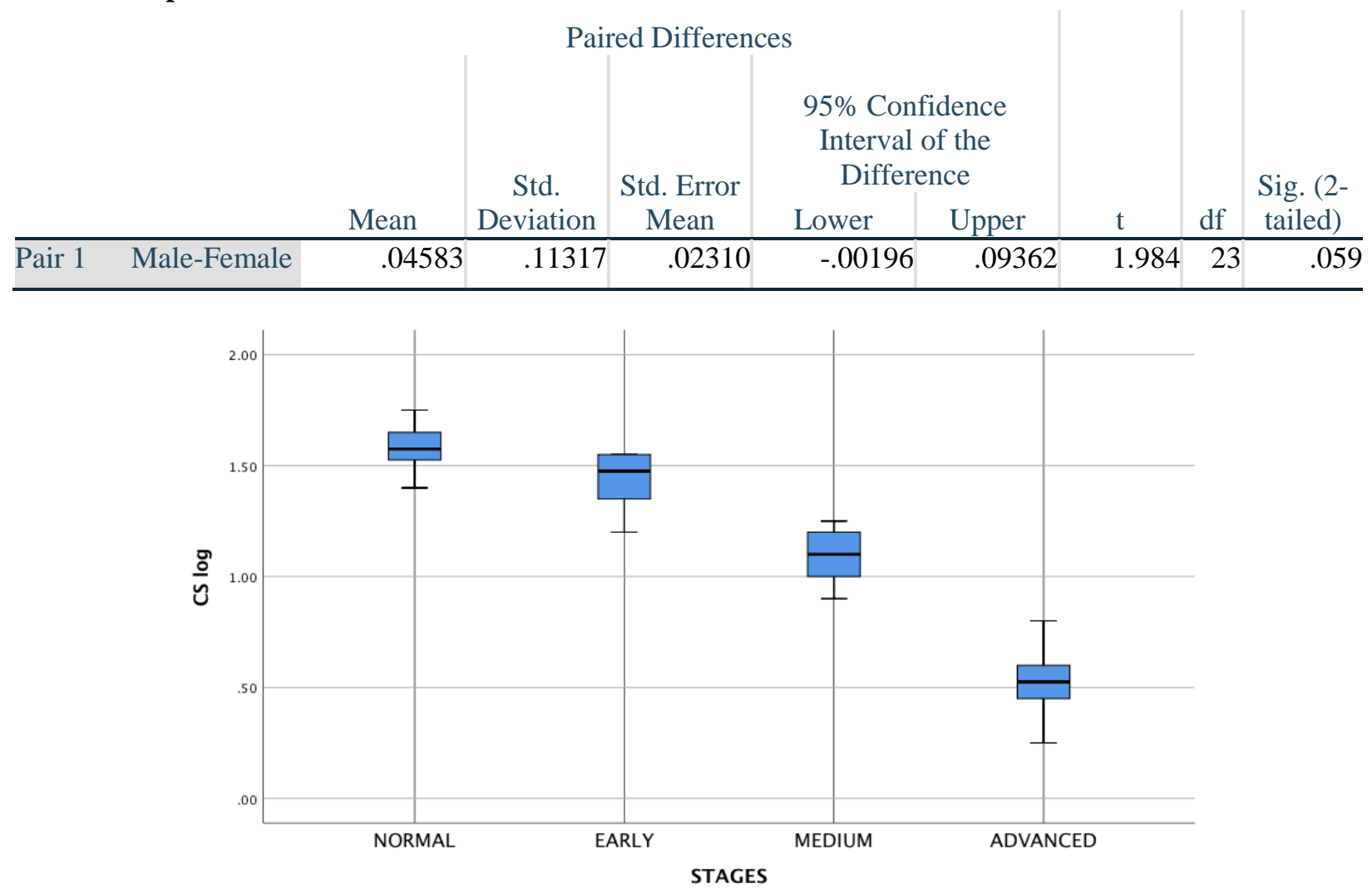

Figure 3: This Box plot illustrates the variance and the decline of the CS log in right eye group due to the AMD presence in simulation mode at all stages. (Normal, Early, Medium, Advanced). 


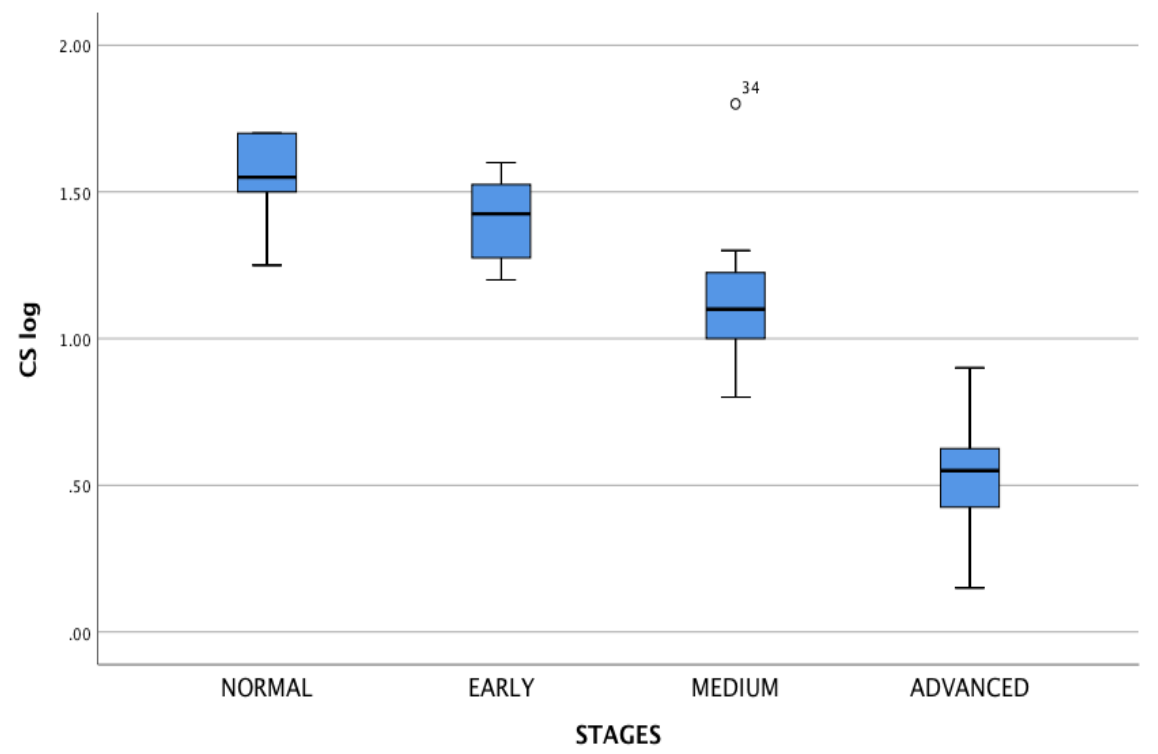

Figure 4: This Box plot illustrates the variance and the decline of the CS log in left eye group due to the AMD presence in simulation mode at all stages. (Normal, Early, Medium, Advanced).One outlier $\left(34^{\text {th }}\right.$ value) was detected that was more than 1,5 box-length from the edge of the box in the boxplot. Inspection of the value did not reveal to be extreme and it was kept in the analysis.

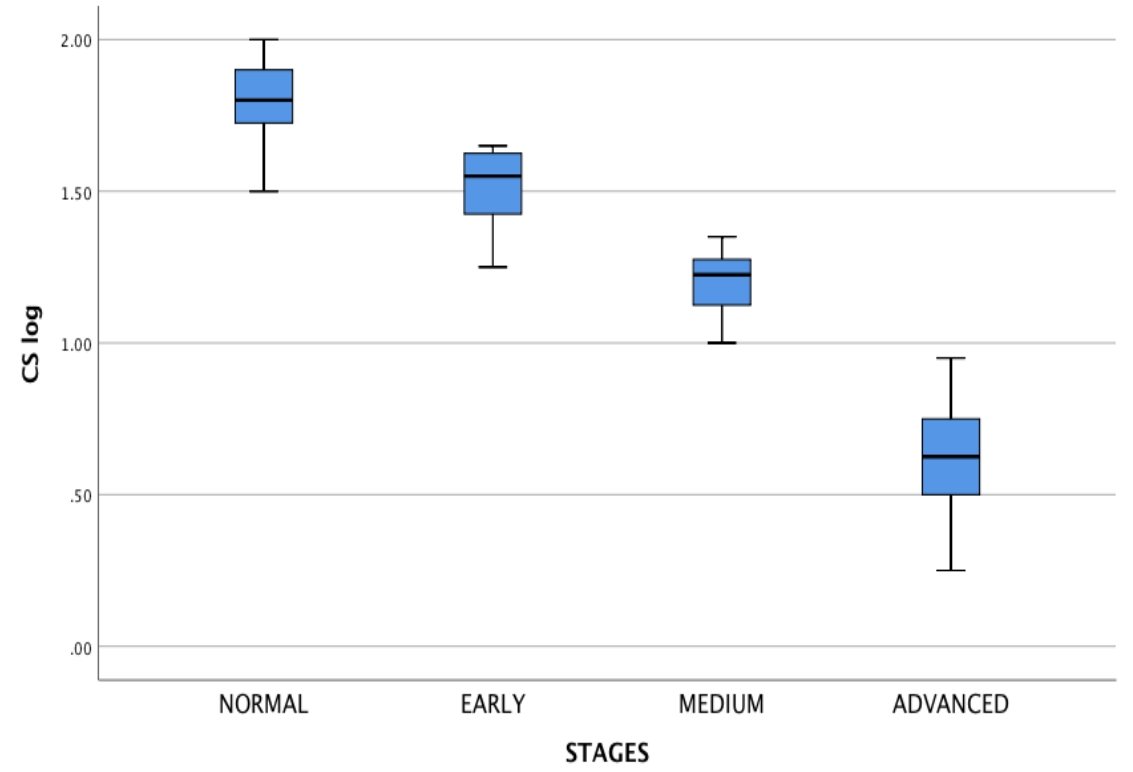

Figure 5: This Box plot illustrates the variance and the decline of the CS log in Binocular group due to the AMD presence in simulation mode at all stages. (Normal, Early, Medium, Advanced).

\section{Discussion}

A one-way ANOVA was conducted to determine if the means was statistically significantly different for different groups. Participants were classified into three groups, right eyes $(n=12)$, left eyes $(n=12) \&$ binocular $(n=12)$ and in three simulation stages ( Early, Medium, Advanced). One outlier was detected that was more than 1.5 box-lengths from the edge of the box in a boxplot. Inspection of the value did not reveal to be extreme and it was kept in the analysis. Data was normally distributed for each group, as assessed by Shapiro-Wilk test ( $\mathrm{p}>0.05$ ) and by Q-Q plots. Data is presented as mean \pm standard deviation. Contrast sensitivity score was statistically significantly different between different groups, Right eyes $\mathrm{F}(3,44)=181.551, \mathrm{p}<0.05, \omega^{2}=0,92$, Left eyes $\mathrm{F}(3,44)=132.189, \mathrm{p}<0.05$, $\omega^{2}=0,90$, Binocular $\mathrm{F}(3,44)=64.074, \mathrm{p}<0.05, \omega^{2}=0,81$. CS score was decreased, from the Normal $(\mathrm{M}=$ $1.57, \mathrm{SD}=0.09)$ to the Early $(\mathrm{M}=1.43, \mathrm{SD}=0.11)$, Medium $(\mathrm{M}=1.09, \mathrm{SD}=0.12)$ and Advanced $(\mathrm{M}=0,51, \mathrm{SD}=$ $0.14)$ Right Eye group, in that order. 
CS score was decreased, from the Normal $(M=1.55, S D=0.13)$ to the Early $(M=1.41, S D=0.14)$, Medium $(M=$ $1.13, \mathrm{SD}=0.25)$ and Advanced $(\mathrm{M}=0,53, \mathrm{SD}=0.22)$ Left Eye group, in that order. $\mathrm{CS}$ score was decreased, from the Normal $(\mathrm{M}=1.80, \mathrm{SD}=0.13)$ to the Early $(\mathrm{M}=1.52, \mathrm{SD}=0.12)$, Medium $(\mathrm{M}=1.20, \mathrm{SD}=0.10)$ and Advanced $(\mathrm{M}=$ $0,61, \mathrm{SD}=0.21)$ Binocular group, in that order.

Turkey post hoc analysis revealed an decrease in CS values in all groups and every stage, which was statistically significant $\mathrm{p}=0.000<0.05, \mathrm{p}=0.005<0.05, \mathrm{p}=0.026<0.05$ with only one exceptions, in Binocular group the decrease from Normal to Early $(0,14,95 \%$ CI, $-0,71$ to 0,35$)$, which was not statistically significant $(\mathrm{p}=0.299)$. A pairedsamples t-test was used to determine whether there was a statistically significant mean difference between Right and Left eyes, $(-0,005 \pm 0,1825)$ and statistically significant increase of $-0,005(95 \% \mathrm{CI},-0.058$ to 0,047$), \mathrm{t}(47)=-0,198$, $\mathrm{p}=0,844>0,05, \mathrm{~d}=-0.027$. There was no statistically significant difference in the means between Right and Left eyes. A paired-samples t-test was used to determine whether there was a statistically significant mean difference between Men and Women, $(0,045 \pm 0,113)$ and a statistically significant increase of 0.045 (95\% CI, -0.001 to 0,093$), t(23)=$ $1.984, p=0,059>0,05, d=0.398$. There was no statistically significant difference in the means between genders.

Normal CS values were compared with contrast sensitivity values in simulated AMD conditions to evaluate the correlation between the AMD presence and CS decline in every stage of the disease. Results demonstrated that contrast sensitivity decreases even in the early stage of AMD. In pathological conditions, the contrast sensitivity is reduced at all spatial frequencies, but a number of different clinical conditions have a selective effect on different areas of the CSF.

\subsection{Conclusions}

The result of the study is significantly useful in addressing the needs of investigation between control and elderly groups, in order to identify its strengths and weaknesses, and the relevance of its competencies to the daily activities . Based on the findings of the study the following conclusions were drawn.

Contrast sensitivity is considered as a necessary diagnostic and prognostic part for the clinical assessment of visual function. It has been recognized in numerous studies that it offers more accurate information about the patient's visual performance. In all stages of AMD simulation, a decreased sensitivity is revealed. The assessment of contrast sensitivity can be a part of medical preventive eye care, furthermore for populations with limited access to public health.

\subsection{Recommendations}

While the CS test is in progress under the AMD simulation effect, the participants in medium and advanced stages have trouble knowing which letter is being tested. So it is clear that clinical groups with central field losssuch as AMD, diabetes maculopathy, amblyopia, cataract have the same difficulty if many letters are present. Therefore recommended to show one letter at a time. The participants before the examination must be rested, intense activities on the same day or the previous without adequate rest will affect the reliability of the data, so it is advisable to postpone the examination. Further, fatigue of participants or memorized answers can increase errors, so the researcher must be ready to acknowledge the participants failure answers, double question for the letter reading is recommended. Future research is proposed in a wider healthy sample over different periods of time to study environmental, hereditary and lifestyle impacts in normal contrast sensitivity values. Finally we conclude the need for contrast sensitivity assessment to be performed in clinical practice to prevent early stages of disease (age-related macular degeneration, cataracts, glaucoma) that do not initially affect visual acuity and are difficult to diagnose in time.

\section{Acknowledgment}

Profound gratitude and appreciation are extended to the department of Biomedical Science at the University of West Attica, Athens, Greece and the head of Postgraduate research group "Investigative Techniques in Optometry", Dr. P. Drakopoulos, for his assistance and supervision of the research.

\section{Declaration of Conflicting Interests}

The authors declared no potential conflicts of interest with respect to the research, authorship, and/or publication of this article.

\section{Funding}

The authors received no financial support for the research, authorship, and/or publication of this article. 


\section{References}

American Academy of Opthalmology. 2017-2018. «Age Related Macular Degeneration and Other Cause of Choroidal Neovascularization.»

Bonastre J., Le Pen C., Soubrane G., Quentel G. (2003). « The burden of age related macular degeneration: results of a cohort study in two French referral centres.» Pharmacoeconomics 181-190.

Campbell F.W. (1983). «Why do we Measure Contrast Sensitivity.» Behavioural Brain Research (elsevier) 10: 87-97.

Colijn J.M. (2017). Prevalence of Age-Related Macular Degeneration in Europe: The Past and the Future. Opthalomology.1753-1763.

David B. Elliot (2007). Assessment of visual function. Clinical procedures in primary eye care. 29-81.

De Jong P.T. Age-related macular degeneration. N Engl J Med. (2006).355(14):1474-1485.

Denis G. Pelli, Peter Bex. 2013. «Measuring contrast sensitivity.» Vision Research 90: 10-14.

Elliot David, David Whitaker, Lorraine Bonette. (1990). «Differences in the legibility of letters at contrast threshold using the Pelli-Robson Chart.» Opthalm.Physiol.Opt, 323-326.

Fechner G.T. 1860/1966. Elemente der Phsychophysik 1860. Leipzig:Breitkopf und Hertel.Translation: H.E.Adler. New York: Holt,Rinehart and Winston.

Fritsche L.G., (2014). Age-related macular degeneration: genetics and biology coming together. Annual Review of Genomics and Human Genetics, 151-171.

Kanski Jack. (2004). Clinical Opthalmology. Athens: Scientific Publications Parisianou SA.

Li J., B.J Tripathi. (2008). «Drug-induced ocular disorders.» Drug Safety:An international Journal of medical Toxicology and drug Experience, 127-141.

Lim Jennifer I. (2013). Age related macular Degeneration. LLC Taylor \& Francis Group. Chicago IL: CRC Press.

Mantyjarvi Maija, Tarja Laitinen. (2001). «Normal Values for the Pelli-Robson contrast sensitivity test.» J. Cataract Refractive Surgery, Vol 27, 26-266.

Margaret M. DeAngelis, L. A. (2017). Genetics of age-related macular degeneration (AMD). Human molecular genetics, R45-R50.

Martin et al., US. Patent 5,737,056, "METHOD FOR SIMULATION OF VISUAL DISABILITIES". 1998.

Pascolini, Donatella \& Paolo Silvio Mariotti. (2012). «Global estimates on visual impairment in the year 2010.» British Journal of Ophthalmology, 614-618.

Pelli Denis G., Bex Peter. (2013). «Measuring contrast sensitivity.»Vision Research, 15 04: 10-14., 2013. «Measuring contrast sensitivity.» Vision Research, 10-14.

Pelli D.G., Farell B. (2010). Psychophysical methods.Vol.III, $\sigma \varepsilon$ Handbook Of Optics:Vision and Optics, C. De Cusatis, J.Enoch,V.Lakshminarayanan,G.Li, C.MacDonald et.al M.Bass, 3.1-3.12. New York: McGraw-Hill.

Pelli D.G., J.G Robson \& A.J Wilikings. (1988). «The design of a new letter chart for measuring contrast sensitivity.» Clinical Vision Sciences 2, 187-199.

Pelli D.G., and B. Farell. (1999). «Why use noise.» Journal of the Optical Society of America, 647-653.

Pelli D.G., and J.G Robson. (1991). «Are letters better than Gratings.» Clinical Vision Sciences, 409-411.

Wong WL. (2014). Global prevalence of age-related macular degeneration and disease burden projection for 2020 and 2040: a systematic review and meta-analysis. Lancet global Health, 106-16.

Wong EYH., Guymer RH., Hassell JB., Keefe JE. (2004). «The experience of age-related macular degeneration.» J. Vis Imp Blind (Elsevier) 629-640. 